\title{
THE GAMMA-RAY OBSERVATORY
}

\author{
DONALD A. KNIFFEN \\ NASA Goddard Space Flight Center, Greenbelt, Maryland 20771
}

\begin{abstract}
The Gamma-Ray Observatory (GRO), a Shuttle-launched free-flying Observatory is currently scheduled for launch in November 1990. The mission provides nearly six orders of magnitude in spectral coverage, from about $50 \mathrm{KeV}$ to about $30 \mathrm{GeV}$, with a sensitivity over the entire range over an order of magnitude greater than that of previous observations. The 16,000 kilogram Observatory contains four instruments on a stabilized platform. The mission duration is expected to be from six to ten years. A Science Support Center has been established at the Goddard Space Flight Center for the purpose of supporting a vigorous Guest Investigator Program.
\end{abstract}

\section{Introduction}

The Gamma-Ray Observatory (GRO) was announced in 1977 as an opportunity for gamma-ray experiments to be included on a free-flying observatory to be launched aboard the space shuttle. The mission was planned as a 3 -axis oriented platform containing four instruments with a planned two year lifetime on orbit. It was later decided that the enhanced science return would justify extending the duration of orbital operations. By the time such an option was adopted, the development of the instruments and spacecraft was well underway. Studies showed that the only consumable quantity clearly preventing a lifetime of from six to ten years was the propellant gas required to maintain the operational orbit against atmospheric drag. GRO was manifested on a high performance Shuttle so that it might be taken directly to its operational altitude of 450 kilometers at the beginning of the mission. This provides sufficient fuel savings to allow the operational orbit to be maintained for up to ten years and still provide fuel for a controlled reentry.

GRO is a $16,000 \mathrm{~kg}$ spacecraft (Figure 1) containing a complement of four instruments to make observations of the gamma-ray sky over the range from about $50 \mathrm{KeV}$ to $30 \mathrm{MeV}$ (Figure 2). This very wide dynamic range requires the use of different instruments with a number of detection techniques. The $\mathrm{Z}$-axis of the spacecraft may be pointed to any region of the sky at any time. However, once chosen, the $\mathrm{X}$-axis must be selected to satisfy sun angle constraints which affect the power from the solar panels and the thermal environment of various spacecraft components. The attitude knowledge will be maintained to an accuracy of 2 arcminutes. Absolute time will be accurate to .1 millisecond.

The extension of the mission lifetime beyond the original two years makes it possible to expand the scientific involvement beyond the original Principal Investigators (PIs) and their Co-Investigators. This not only increases the access to the data and observing opportunities, but it also enhances the scientific return by the infusion of ideas from a broader community. However, since the data analysis systems were designed for a PI class mode of operation, a program to involve those from outside the PI teams must be done in a manner that carefully considers the 


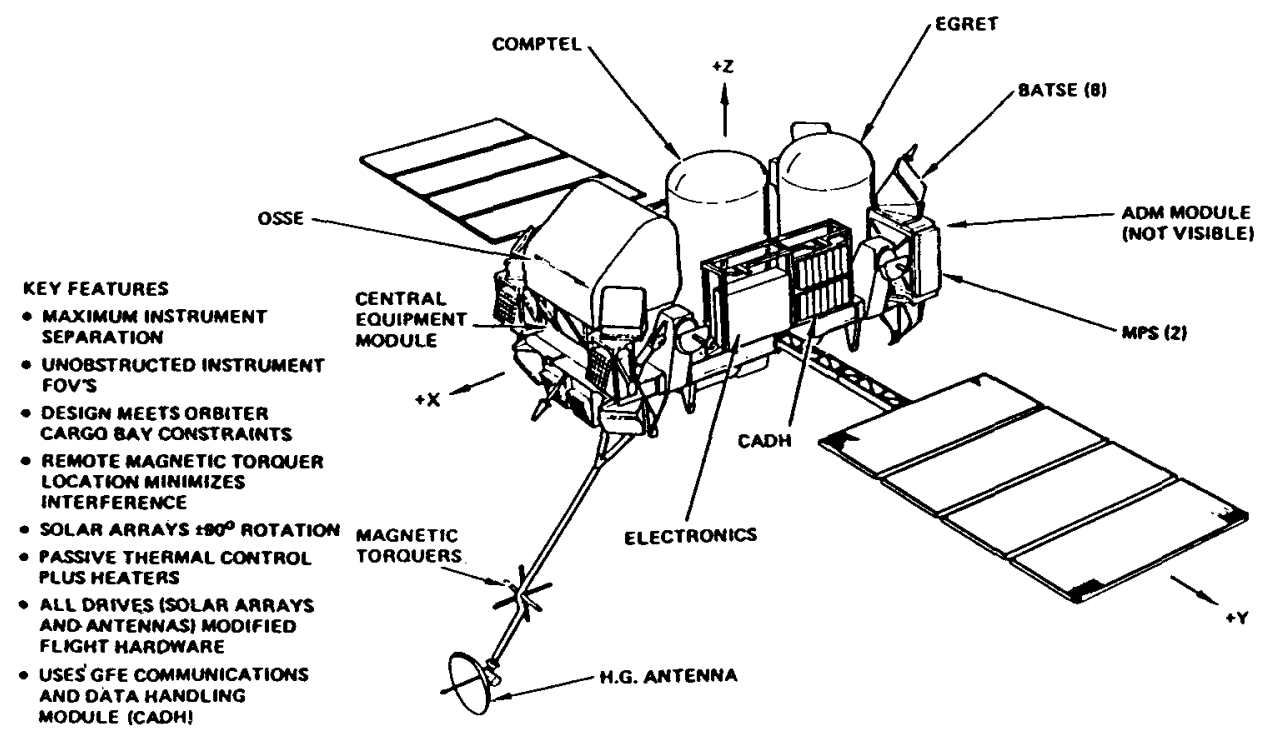

Fig. 1. The Gamma-Ray Observatory.

difficulties in analyzing and interpreting the data. The GRO Science Support Center has been established at the Goddard Space Flight Center to assist the Guest Investigators in accessing the GRO data.

\section{The Instruments}

Figure 2 shows the spectral coverage of the four instruments. They are the Burst and Transient Source Experiment (BATSE), the Imaging Compton Telescope (COMPTEL), the High Energy Telescope (EGRET) and the Oriented Scintillation Spectrometer Experiment (OSSE). The BATSE experiment is designed to monitor the entire sky continuously for bursts and transient gamma-ray events using eight uncollimated, wide-field detectors placed at all corners of the spacecraft, top and bottom. The OSSE experiment, with a relatively narrow field of view, will primarily be sensitive to discrete sources, the dominant feature of the low energy gamma-ray sky. The COMPTEL and EGRET experiments are wide field instruments designed to provide a high sensitivity survey of the medium and high energy gamma-ray sky.

\section{BATSE}

BATSE is optimized to measure brightness variations on timescales down to milliseconds. To accomplish this eight detector modules of identical configuration are used. The main detector is a large area sodium iodide scintillation crystal ( 20 inches in diameter by one-half inch thick). Light produced as the incident gamma rays interact in this scintillator are sensed by three large photomultiplier tubes placed behind it, while in front is a plastic scintillator used to reduce the background due 


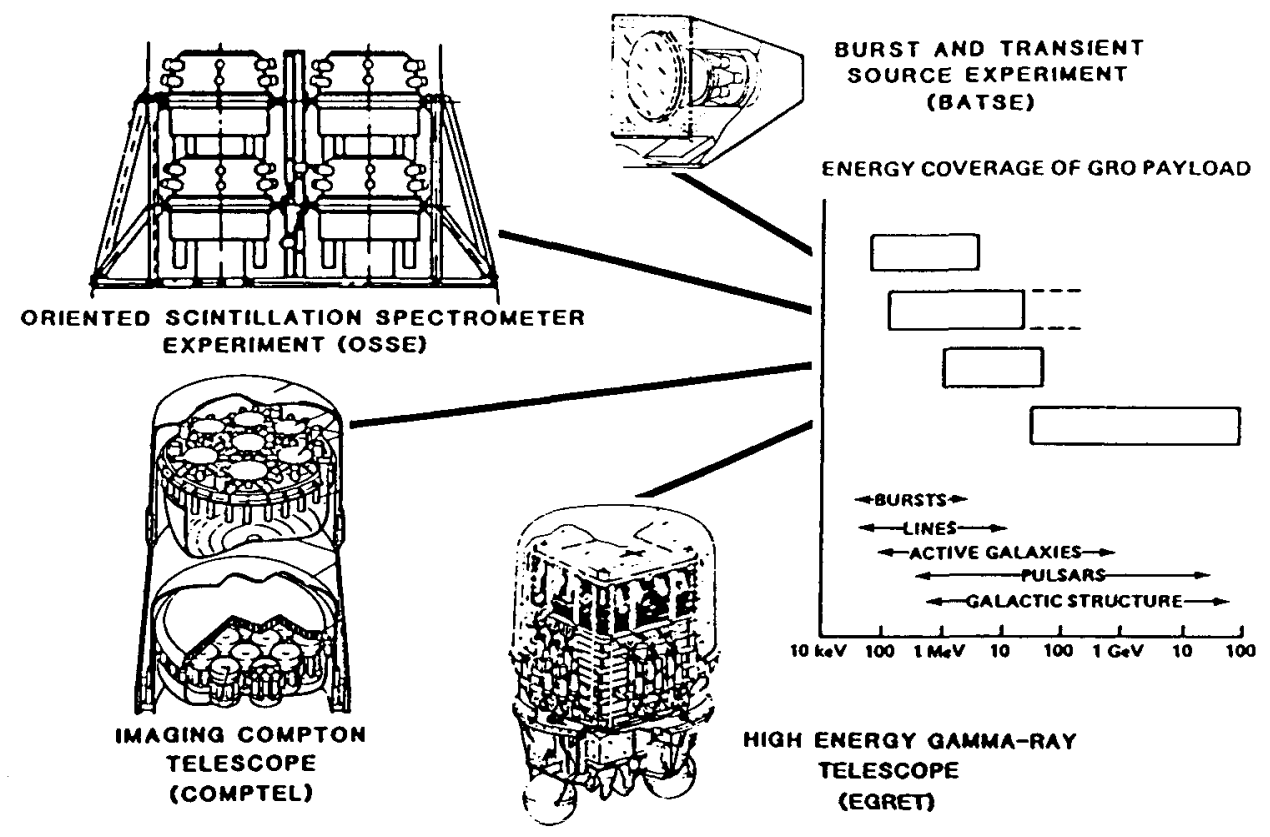

Fig. 2. Energy Coverage of the GRO Experiments.

to charged particles. Gamma-rays are inclined to pass through plastic without interaction. Upon detection of a fast, intense transient event, such as a gamma-ray burst, the BATSE processes large amounts of gamma-ray data for later transmission to the ground. It also sends a signal to the three other telescopes on the GRO that such an event has occurred so that it can be studied in greater detail over a wider energy band. A smaller spectroscopy detector, optimized for broad energy coverage and good resolution is contained with each of the 8 detectors.

Dr. Gerald R. Fishman of the NASA Marshall Space Flight Center (MSFC) is the Principal Investigator of the BATSE experiment with Co-Investigators at MSFC, The University of Alabama, Huntsville, the Goddard Space Flight Center (GSFC) and the University of California, San Diego.

\section{OSSE}

OSSE has been designed to undertake comprehensive observations of astrophysical sources in the 0.1 to $10 \mathrm{MeV}$ range, and also includes limited capability above $10 \mathrm{MeV}$, primarily for solar gamma-ray and neutron observations. The instrument consists of four identical detector systems, each of which is articulated to provide a 192-degree rotation capability about the $\mathrm{Y}$-axis of the spacecraft. Normally, two detectors will observe the source, and two a nearby off-source region. The combination will be reversed at regular intervals, and the difference signal represents the net source flux. Each detector system includes a 33-cm diameter NaI-CsI phoswich assembly which provides the basic gamma-ray detection capability. Active shield- 
ing is provided by an annular NaI shield and the CsI section of the phoswich. The acceptance aperture of $3.8 \times 11.4$ degrees is defined by a passive tungsten alloy collimator. The offset pointing feature also allows the observation of targets away from the prime viewing axis of GRO. This permits viewing secondary sources when the main spacecraft axis is occulted by the earth, and allows for quick response observations of transient phenomena such as solar flares and novae without impacting the Observatory viewing program in a major way.

Dr. James D. Kurfess of the U.S. Naval Research Laboratory (NRL) is the Principal Investigator of the OSSE experiment with Co-Investigators at NRL, Northwestern University, Clemson University and the Royal Aircraft Establishment.

\section{COMPTEL}

In COMPTEL, gamma rays are detected by the occurrence of two successive interactions: the first one is a Compton collision in a detector of low- $\mathrm{Z}$ material (liquid scintillator) followed by a second interaction in a detector with high-z material (NaI), in which the scattered gamma-ray is totally absorbed. The sequence of collisions is confirmed by a time-of-flight measurement between the two detectors. The neutron induced background events, which may simulate double scatter gamma-ray events, are identified and rejected on the basis of the shape of the light pulse emitted in the first scatter detector. This method, in combination with effective charged particle shield detectors is very effective in suppressing the otherwise inherent instrumental background. The spatial resolution in the two detectors, together with the well defined kinematics of the Compton interaction, permits the reconstruction of discrete and extended sky images over a wide field of view with a resolution of the order of a degree. The minimum source detectability that can be achieved with COMPTEL is a few percent of the total emission from the Crab, the standard candle of gamma-ray astronomy. The first or upper detector of COMPTEL contains 7 cylindrical modules of liquid scintillator. Each module is approximately $28 \mathrm{~cm}$ in diameter and $8.5 \mathrm{~cm}$ thick and viewed by eight photomultipliers. The second detector consists of 14 cylindrical NaI blocks of $7.5 \mathrm{~cm}$ thickness and $28 \mathrm{~cm}$ diameter. Each block is viewed by seven photomultipliers from below. The distance between the first and second detectors is 1.50 meters. Each detector is surrounded by a thin anticoincidence shield of plastic scintillator. The Principal Investigator for the COMPTEL instrument is Dr. Volker Schoenfelder of the Max-Planck Institute for Extraterrestrial Physics (MPE) in Garching, West Germany. Co-Investigators are located at MPE, at the Laboratory for Space Research, Leiden, at the Space Sciences Department of the European Space Agency, and at the University of New Hampshire.

\section{EGRET}

The high energy instrument on GRO, EGRET covers the broadest range, from about $20 \mathrm{MeV}$ to about $30,000 \mathrm{MeV}$. Like COMPTEL it is an instrument with a wide field, good angular resolution and very low background. Because it is designed for the study of higher energy gamma-rays, the detector is optimized to detect 
gamma-rays when they interact by the dominant high energy process which forms an electron and a positron. This unique signature allows a positive identification of the parent gamma-ray. The basic imaging portion of this instrument consists of two sections of spark chambers separated by a layer of segmented plastic scintillators. The upper section consists of 28 spark chamber modules interleaved with 27 thin foils of high- $Z$ target material for the conversion of the gamma-rays to an electronpositron pair. After the electrons are formed their trajectory is followed through the remainder of the upper and lower spark chamber sections and into the large $\mathrm{NaI}$ crystal where the rest of their energy is deposited. The NaI counter will provide good precision in estimating the energy of the gamma-ray over the large sensitive range of this instrument. A central scintillator matrix, in coincidence with a similar layer below the bottom spark chamber section, identifies the presence of the electrons, applies the high voltage to the spark chambers and initiates the read-out of the digitized "picture" of the particle trajectories. A large plastic scintillator dome over the instrument identifies and rejects events containing particles which are already charged on entry into the detector.

Co-Principal Investigators of the EGRET instrument are Dr. Carl E. Fichtel of GSFC, Dr. Robert Hofstadter of Stanford University and Dr. Klaus Pinkau of the Max-Planck Institute in West Germany. Co-Investigators are located at each of these institutions and at the Grumman Aerospace Corporation.

Major characteristics and capabilities of the GRO instrument complement are summarized in Table I.

\section{The Gamma-Ray Observatory Mission}

The Gamma-Ray Observatory is now in launch preparations at the Kennedy Space Center in Florida. The launch is currently scheduled for the beginning of 1991, aboard the Space Shuttle Atlantis. Although the orbital altitude is degraded by atmospheric drag, it will be maintained between 440 and $450 \mathrm{~km}$ (Figure 3) by the on-board propulsion system. At the end of the mission the spacecraft will either be retrieved by the Space Shuttle, or will reenter in a controlled manner.

During the orbit operations data will be recorded at a 32 kilobit per second data rate, and these data will be telemetered via the Tracking and Data Relay Satellite (TDRS) once every two orbits at a 512 kilobit rate. Uplink commanding of both the experiments and the spacecraft will also be sent during these TDRS contacts. The mission operations profile are indicated in Figure 4. The Control Center will be at the Goddard Space Flight Center. Data will be received in packetized form with all ancillary information required to analyze the data already inserted on board the spacecraft. Time ordering, overlap elimination and quality checks will be done on ground before the data are forwarded, within 48 hours of receipt, to the experiment data analysis facility sites.

The viewing program for the first 15 months of operation is currently in the final process of development by the GRO Timeline Committee and will be designed to provide a nearly uniform full sky survey of two week exposures for the two wide field instruments (EGRET and COMPTEL). The narrow aperture instrument (OSSE)instrument will select 30 primary and secondary targets for discrete source 


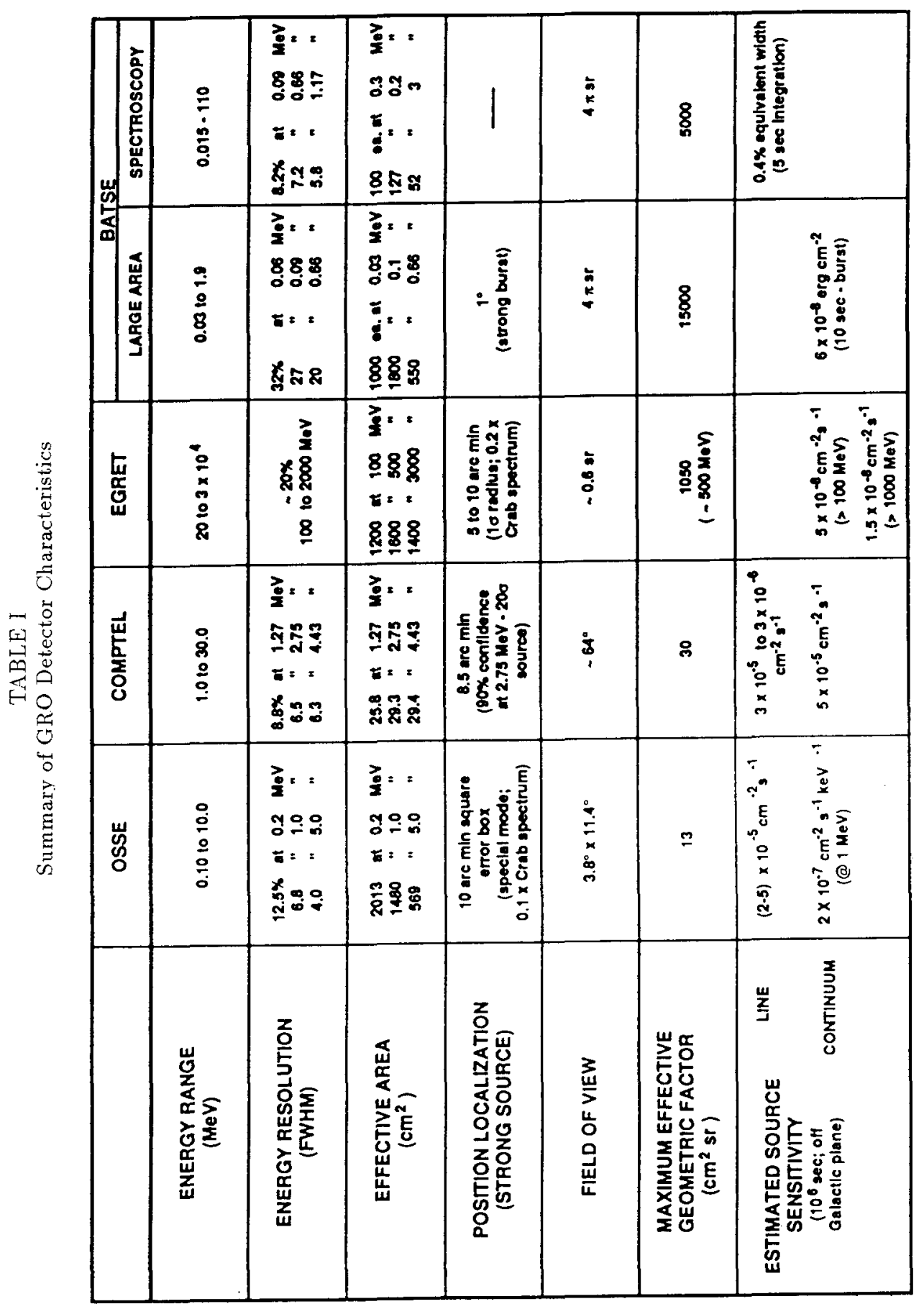




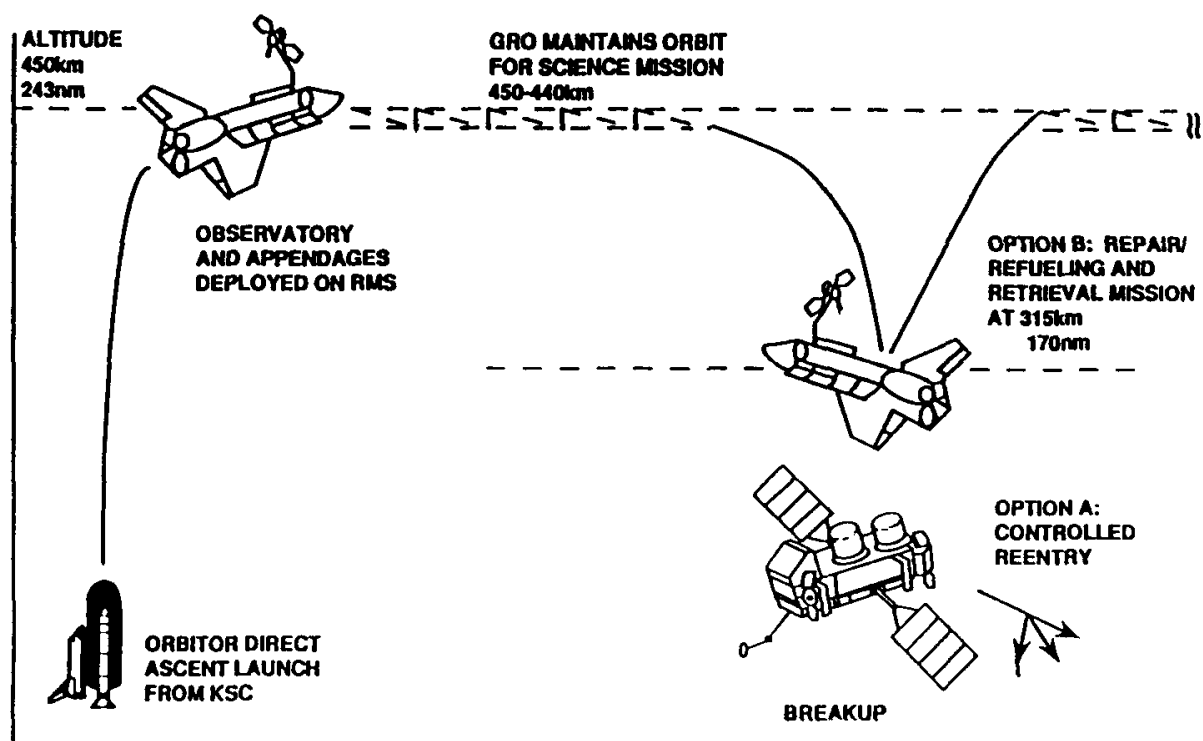

TIME

Fig. 3. The GRO Mission Profile.

studies during this 15 month period and the burst instrument (BATSE) covers the entire unocculted sky at all times. The viewing program for following years will be determined later, but will emphasize deep searches and the study of source time variations on many timescales. The timing of the NASA Research

Announcement for the Guest Investigator Participation will be chosen so that observations proposed by selected Guest Investigators will be included in the observing plan as efficiently as possible. A considerable effort has bee placed on coordinating with both space- and earth-based observations at other wavelengths to enhance the value of such observations at all wavelengths.

\section{The Science Support Center}

The central point of contact for Guest Investigator support will be the GRO Science Support Center (GROSSC), the Goddard Space Flight Center. In addition to its function of aiding Guest Investigators in accessing GRO data the GROSSC will be a source of information for potential users at all stages of their involvement, from the preproposal stage, through the analysis phase. The Center will provide information on the availability and timing of opportunities, and give technical support in the implementation of chosen guest investigations. It will be the source for technical information on the GRO instruments, for scientific and technical information on the GRO spacecraft and mission, for catalogs of data on GRO and other astrophysics and astronomy observations, for the status of GRO observations, for availability of useful analysis software, and other such information of use for GRO investigations. 


\section{Summary}

The Gamma-Ray Observatory represents a dramatic step in capability at the highest energy region of the electromagnetic spectrum accessible by space platforms. Many exciting advances in our knowledge of our explosive universe can be predicted based on the pioneering work of many previous missions with instruments with narrower objectives. The Space Shuttle brings the opportunity to make these advances and the many unpredictable discoveries that result when such dramatic increases in observing capability occur. With the next decade should bring great advances in astrophysics. 